\title{
Fundamental Properties of Infilled Concrete for Hollow PC
}

\author{
Guncheol LEE \\ Dept. Architectural Engineering \\ Korea National University of Transportation \\ Chungju, Republic of Korea \\ e-mail: gclee@ut.ac.kr \\ Jaeyup KIM \\ Dept. Architectural Engineering \\ Korea National University of Transportation \\ Chungju, Republic of Korea \\ e-mail: kimjy67@ut.ac.kr

\section{Jiwoong PARK} \\ Dept. Architectural Engineering \\ Korea National University of Transportation \\ Chungju, Republic of Korea \\ e-mail: jiwoong9203@naver.com
}

\author{
Sooyeon SEO \\ Dept. Architectural Engineering \\ Korea National University of Transportation \\ Chungju, Republic of Korea \\ e-mail: syseo@ut.ac.kr \\ Gao SHAN \\ Dept. Architectural Engineering \\ Korea National University of Transportation \\ Chungju, Republic of Korea \\ e-mail: gaoshan0831@naver.com \\ Kidon SHIN \\ Dept. Architectural Engineering \\ Korea National University of Transportation \\ Chungju, Republic of Korea \\ e-mail: sgd0313@naver.com
}

\begin{abstract}
The purpose of this study is to find the basic characteristics of HPC consolidating concrete. The results are presented as follows: In terms of compression strength, the strength of BS mixed with Alkaline Activator (AA) was stronger than that of general BS. It is judged that Shrinkage Reducing Agent (SRA) considerably less influences compression strength. In terms of length change, the length of BS mixed with the AA changed smallest. It was found that the length change reduced further with the use of SRA.
\end{abstract}

Keywords-hollow PC; length change; shrinkage reducing agent; alkaline activator

\section{INTRODUCTION}

The current construction market features high-rise buildings, large buildings, and complex buildings. As a result, there are more demands of the buildings with a large span and story height. As reinforced concrete construction technique is applied to the buildings, there are more temporary works like formwork which cause a limit to a shortened construction period. PC method for supplementation has low economy because of increased joint portions and need to secure structural performance of joints. In addition, compared to Reinforced Concrete method, the PC method one has weak connection and some problems like insufficient concrete filling. To overcome the problems, Hollow PC (HPC) that improves construction performance of connections, secures integrity and has a hollow generated by centrifugal force making are combined with and cast-inplace concrete. Therefore, a combined HPC column is developed and is studied for commercialization. In the mean time, general concrete is cast in HPC, and after integration a column section is generated. However, drying shrinkage occurs in the cast concrete of HPC, it is possible to make integration of a column section. It can be one of causes that deteriorates the mechanical characteristics of HPC.

Therefore, this study tries to review the shrinkage properties and improvement direction of the concrete filled in HPC and thereby to provide a reference material for improving the mechanical characteristics of HPC.(Reference to Figure 1,2)

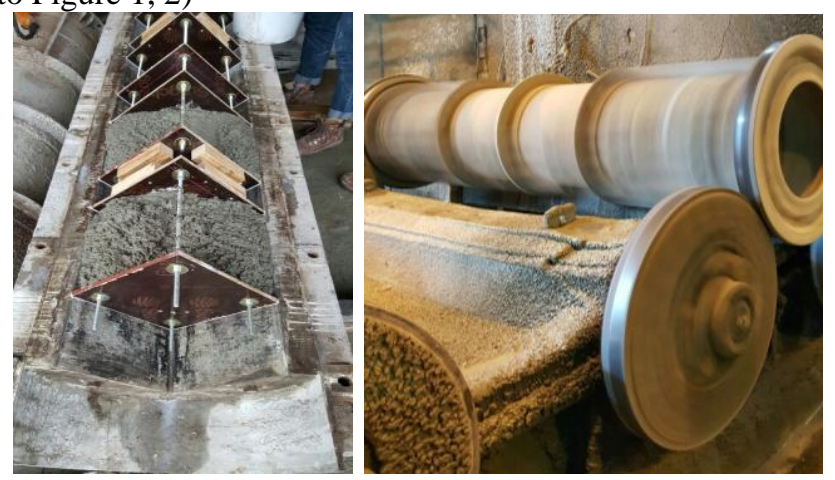

Figure 1. Production process of HPC specimen 


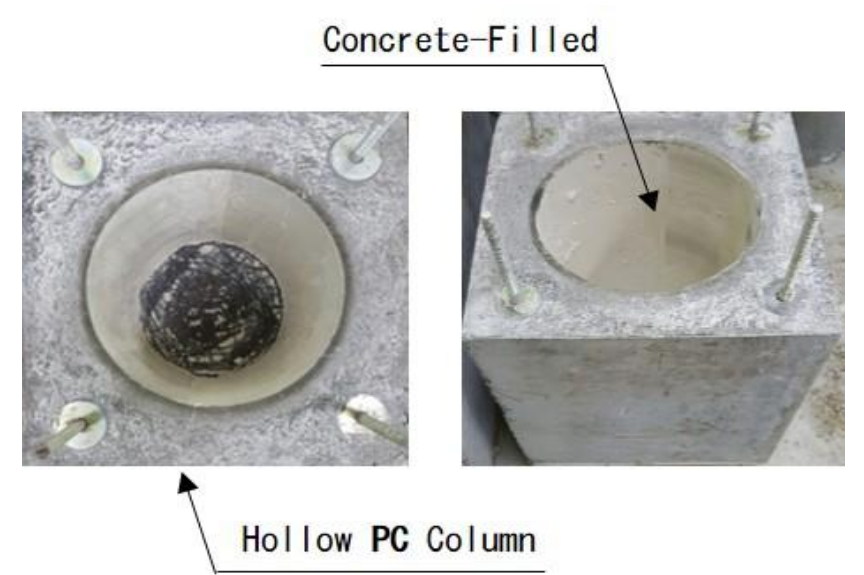

Figure 2. Centrifugal molding HPC

\section{EXPERIMENTAL OUTLINE}

\section{A. Experimental Plans}

The experimental design of this study is presented in Table 1. A target strength was $27 \mathrm{MPa}$ types of powder were OPC, 60\% substituted Blast furnace Slag at mass ratio, BS (AA) mixed with alkaline activator, and each BS case mixed with SRA. At this time, in terms of mass, powder typed alkaline activator had $1.5 \%$ of Blast furnace Slag and $1 \%$ of SRA powder (Binder) mixed. During testing, compression strength and length change were measured, and SEM photographing was performed.

TABLE I. EXPERIMENTAL PLANS

\begin{tabular}{|c|c|c|}
\hline Experimental factors & \multicolumn{2}{|c|}{ Test level } \\
\hline Target strength (MPa) & 1 & 27 \\
\hline Powder type & 6 & $\begin{array}{c}\text { OPC, BS, BS(AA) } \\
\text { OPC-SR, BS-SR, BS(AA)-SR }\end{array}$ \\
\hline BS Replacement ratio (\%) & 1 & 60 \\
\hline AA Replacement ratio (\%) & 1 & 1.5 \\
\hline SRA Replacement ratio (\%) & 1 & 1.0 \\
\hline Experimental details & 3 & $\begin{array}{c}\text { Compressive Strength, } \\
\text { Length change, SEM }\end{array}$ \\
\hline \multicolumn{2}{|c|}{ BS:blast furnace slag, AA:alkaline activator, } \\
SRA:shrinkage reducing admixtures
\end{tabular}

\section{B. Materials}

The basic properties of the powder (Binder) and alkaline activator used in this study are presented in Tables 2 4. Ordinary Portland Cement (OPC) of Korean S and Blast furnace Slag of $\mathrm{H}$ were used. As an alkaline activator, Modified Alkali Sulfate of was used.
TABLE II. PHYSICAL AND CHEMICAL PROPERTIES OF BINDER

\begin{tabular}{|c|c|c|c|c|c|}
\hline \multirow{2}{*}{} & \multirow{2}{*}{$\begin{array}{c}\text { Density } \\
\left(\mathrm{g} / \mathrm{cm}^{3}\right)\end{array}$} & \multirow{2}{*}{$\begin{array}{c}\text { Fineness } \\
\left(\mathrm{cm}^{3} / \mathrm{g}\right)\end{array}$} & \multicolumn{3}{|c|}{ Chemical Composition (\%) } \\
\cline { 4 - 6 } & 3.14 & 3,390 & 3.46 & 2.62 & 1.87 \\
\hline $\mathrm{OPC}$ & 2.91 & 4,370 & 5.80 & 2.36 & 0.37 \\
\hline $\mathrm{BS}$ & 3.03 & 4,060 & 3.12 & 2.52 & 2.62 \\
\hline BS(AA) & $3.03 \mathrm{SO}^{3}$ & $\begin{array}{c}\text { Ignition } \\
\text { Loss }\end{array}$ \\
\hline
\end{tabular}

TABLE III. Physical Properties of Alkaline Activator

\begin{tabular}{|c|c|c|}
\hline Type & Image & $\begin{array}{c}\text { Density } \\
\left(\mathrm{g} / \mathrm{cm}^{3}\right)\end{array}$ \\
\hline $\begin{array}{c}\text { Modified Alkali } \\
\text { Sulfate }\end{array}$ & $\begin{array}{c}\text { Light gray inorganic } \\
\text { Powder }\end{array}$ & 2.75 \\
\hline
\end{tabular}

TABLE IV. Physical PROPERTIES OF SRA

\begin{tabular}{|c|c|c|}
\hline Image & Density $\left(\mathrm{g} / \mathrm{cm}^{3}\right)$ & $\mathrm{pH}$ \\
\hline Light gray inorganic Powder & 2.75 & - \\
\hline Liquid Type & 0.950 & 6.0 \\
\hline
\end{tabular}

\section{Experiment Methods}

Testing compression strength and length change was carried out in accordance with Korean Industrial Standards. To measure a change in length, a buried-typed gauge was applied. For SEM photographing, OOO equipment of OOO company was used to observe the microstructure of sample surface. Photo specimens produced for the length change measure is as figure 3 .

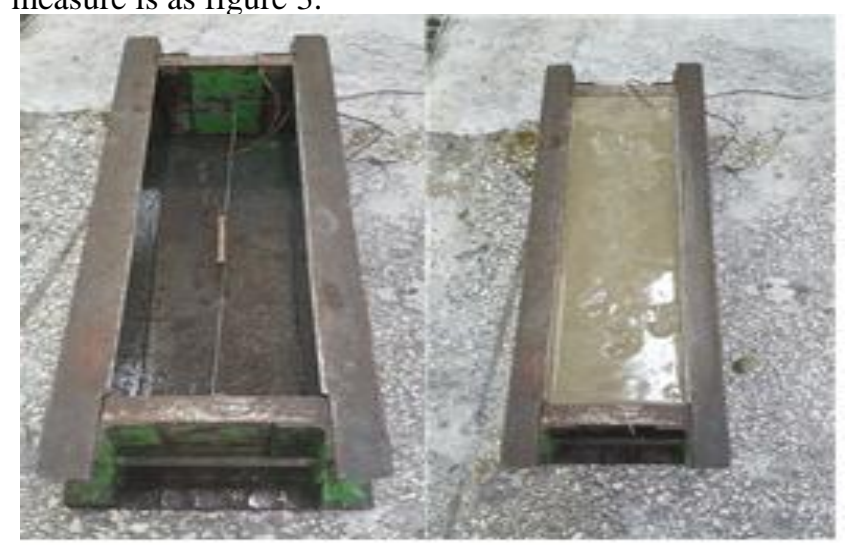

Figure 3. Production process of HPC specimen 


\section{RESULT \& ANALYSIS}

\section{A. Compressive Strength \& SEM Analysis}

Fig. 4 shows different compression strength depending on whether SRA is used. Generally, there was almost no difference in compression strength depending on whether SRA is used. However, regarding types of powder, the specimens with OPC and BS (AA) powder had relatively strong compression strength, whereas the specimen with BS powder had weak compression strength. In particular, in the case of 3-day age, the Blast furnace Slag specimen (BS (AA)) mixed with alkaline activator (AA) had a strength value similar to that of OPC. As shown in Fig.5, it is judged that the addition of alkaline activator in BS powder leads to activating the generation of Ettringite. Also when associate compressive strength results and the SEM photo (1-day age), generation of 에 has been created BS (AA) more than BS a lot, so Early-Age strength analyzed highly determined. Seeing the value of Ettringite when inserted SRA, compared to BS, generation of hydrates is faster than OPC, BS (AA), so it determined that effect of Early-Age strength increase.

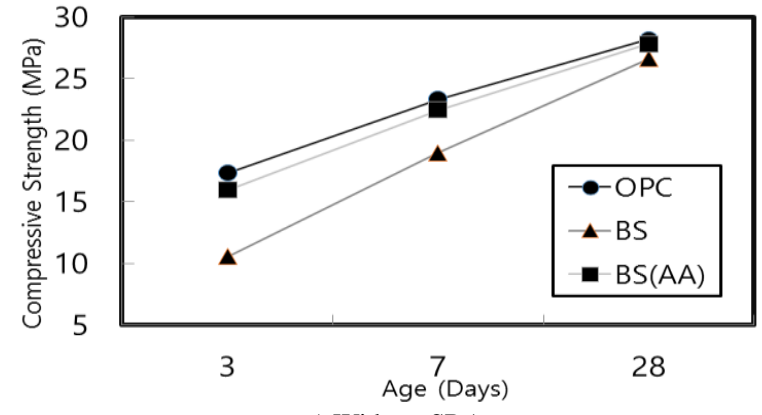

a) Without SRA

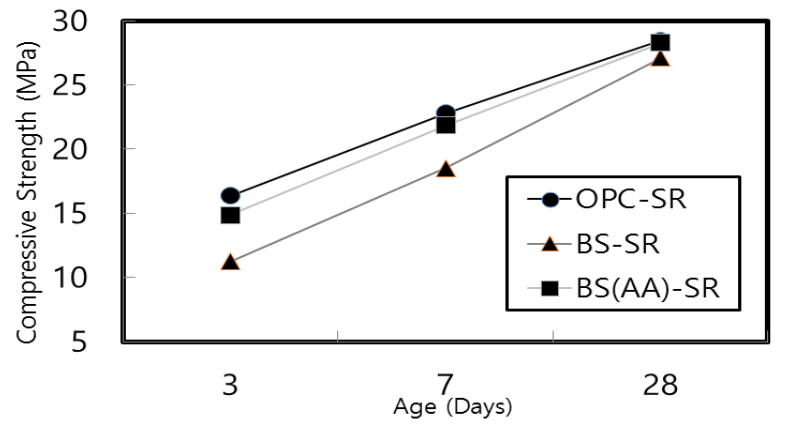

b) With SRA

Figure 4. Compressive Strength Properties

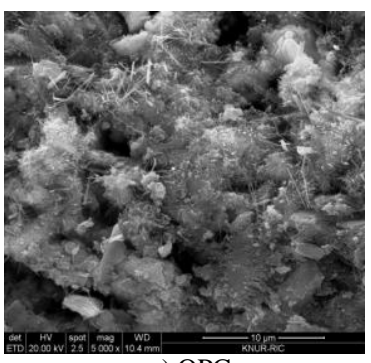

a) OPC

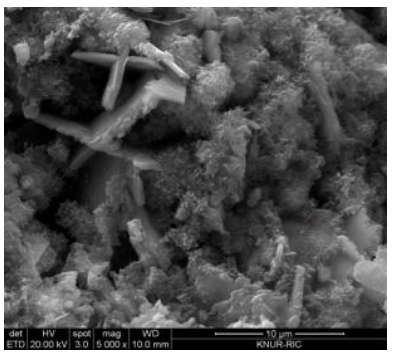

b) BS

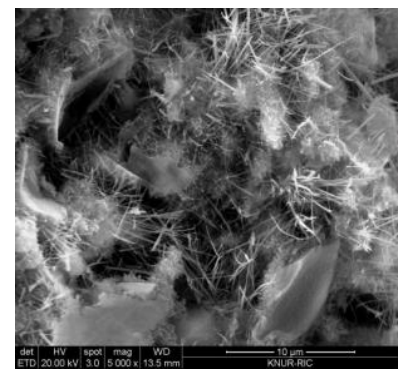

c) BS (AA)

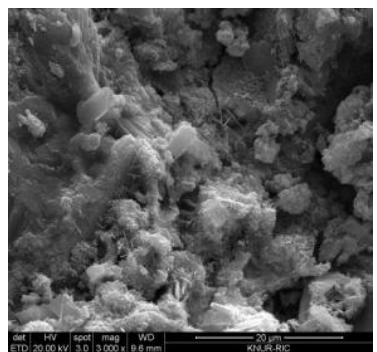

d) OPC-SR

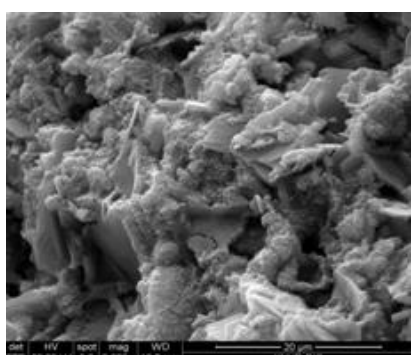

e) BS-SR

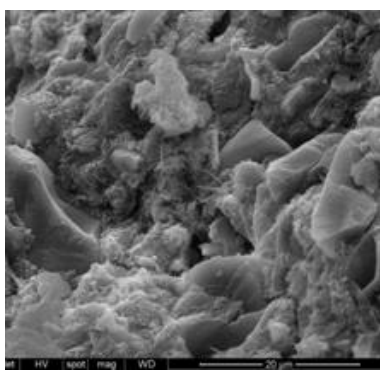

f) $\mathrm{BS}(\mathrm{AA})-\mathrm{SR}$
Figure 5. SEM photo

\section{B. Length Change Properties}

Fig.6 illustrates a change in length depending on whether SRA is mixed. Generally, with a rise in age, the length change rate increased. The case without SRA (a) had the range of 530 480 $\times 10-6$ on the basis of 28-day age. BS (AA) had the lowest, and BS had the highest.

The case with SRA had the range of 490 325 $\times 10-6$ on the basis of 28-day age. In particular, the cases of BS (AA) and BS had a smaller reduction in length change as SRA was used. In general, SRA inserted to control capillary tension, in case of changing pore structure so it is hard to compared as hydrates. With the SEM pictures, it is judged to be hard to analyze the cause. Instead, it is considered to analyze micro pores. 


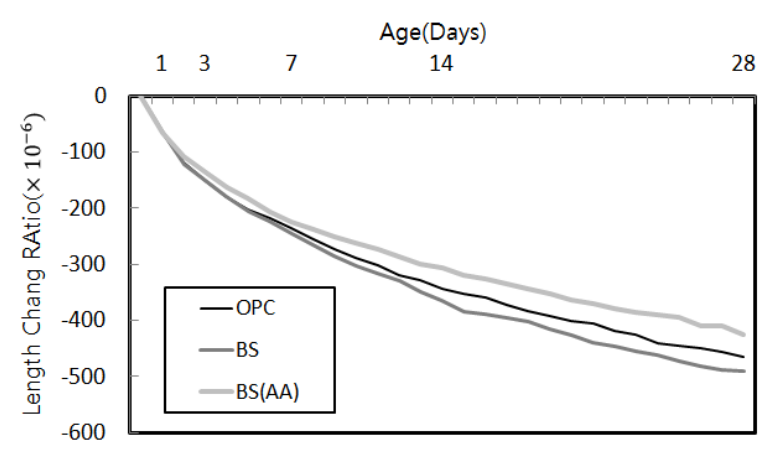

a) Without SRA

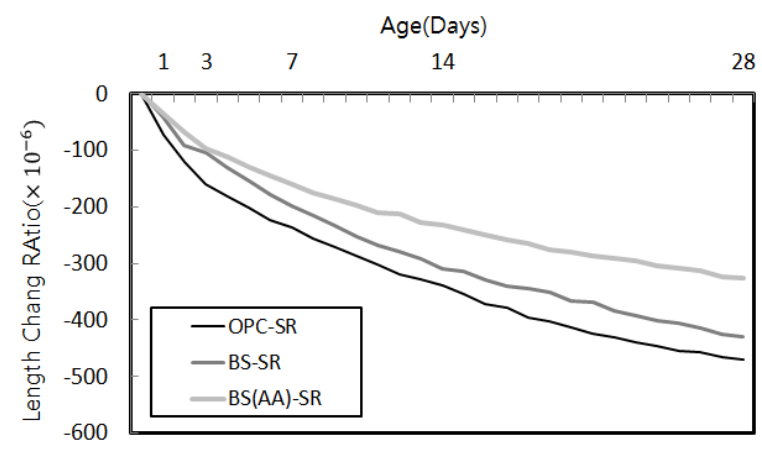

b) With SRA

Figure 6. Length change properties

\section{CONCLUSION}

This study reviewed the shrinkage characteristics of the concrete filled in HPC, and the results are presented as follows.

\section{A. Compressive Strength \& SEM Analysis}

There was no big difference in compression strength depending on whether SRA was mixed. However, when Blast furnace Slag was applied, the initial strength reduced remarkably. Mixing with alkaline activator was found to improve the initial strength noticeably. Seeing the value of Ettringite when inserted SRA, compared to BS, generation of hydrates is faster than OPC, BS(AA), so it determined that effect of Early-Age strength increase.

\section{B. Length Change Properties}

When SRA was not mixed, the specimen using Blast furnace Slag (BS) had the largest length change. However, the cases of BS (AA) and BS mixed with SRA had a considerably more reduction in length change than the cases without SRA.

\section{ACKNOWLEDGMENT}

This work was supported by the Human Resource Training Program for Regional Innovation and Creativity through the Ministry of Education and National Research Foundation of Korea (NRF-2015H1C1A1035953)

\section{REFERENCES}

[1] D.W Ryu and 3 others, "An Experimental Study on the Carbonation and Drying Shrinkage of Concrete Using High Volumes of Ground Granulated Blast-furnace Slag", Journal of the Korea Institute of Building Construction, Vol.12 No4 (2012).

[2] S.H Lee and 2 others, "The Effect on Latent Hydraulic Property of the Blast-furnace Slag by Alkali Activator", Journal of the Korea Concrete Institute, Vol.2001 No.5 (2001).

[3] E.H Joo and 5 others, "A Study on the Compressive Strength and Drying Shrinkage of Concrete Depending on Mineral Admixture Kinds", Journal of the Korea Concrete Institute, Vol.2005 No.5 (2005). 